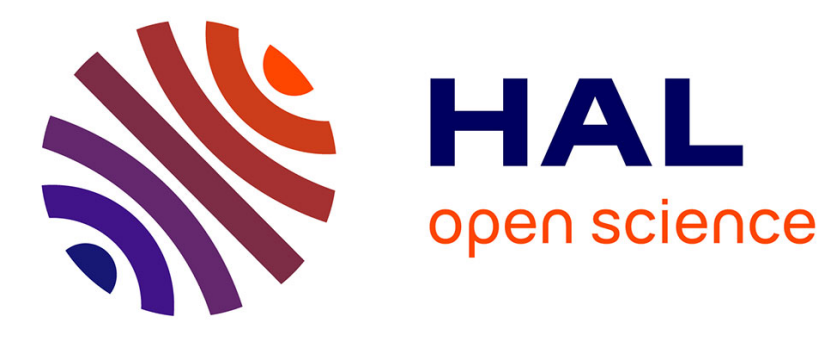

\title{
Quantitative Study of Interface/Interphase in Epoxy/Graphene-Based Nanocomposites by Combining STEM and EELS
}

Yu Liu, Ann-Lenaig Hamon, Paul Haghi-Ashtiani, Thomas Reiss, Benhui Fan, Delong He, Jinbo Bai

\section{- To cite this version:}

Yu Liu, Ann-Lenaig Hamon, Paul Haghi-Ashtiani, Thomas Reiss, Benhui Fan, et al.. Quantitative Study of Interface/Interphase in Epoxy/Graphene-Based Nanocomposites by Combining STEM and EELS. ACS Applied Materials \& Interfaces, 2016, 8 (49), pp.34151-34158. 10.1021/acsami.6b12915 . hal-01835458

\section{HAL Id: hal-01835458 https://hal.science/hal-01835458}

Submitted on 20 Apr 2020

HAL is a multi-disciplinary open access archive for the deposit and dissemination of scientific research documents, whether they are published or not. The documents may come from teaching and research institutions in France or abroad, or from public or private research centers.
L'archive ouverte pluridisciplinaire HAL, est destinée au dépôt et à la diffusion de documents scientifiques de niveau recherche, publiés ou non, émanant des établissements d'enseignement et de recherche français ou étrangers, des laboratoires publics ou privés. 


\title{
Quantitative Study of Interface/Interphase in Epoxy/Graphene-based Nanocomposites by Combining STEM and EELS
}

Yu LIU, Ann-Lenaig HAMON*, Paul HAGHI-ASHTIANI, Thomas REISS, Benhui FAN, Delong HE and Jinbo BAI*

Laboratoire Mécanique des Sols, Structures et Matériaux (MSSMat), CNRS UMR 8579, CentraleSupélec, Université Paris-Saclay, Grande Voie des Vignes, 92290, Chatenay-Malabry, France

Corresponding author: Jinbo BAI, jinbo.bai@centralesupelec.fr

Tel./Fax: +33(0)141131316

Ann-Lenaig HAMON, ann-lenaig.hamon@ centralesupelec.fr

\begin{abstract}
A quantitative study of the interphase and interface of graphene nano-platelets (GNPs) / epoxy and graphene oxide (GO)/ epoxy was carried out by combining scanning transmission electron microscopy (STEM) and electron energy-loss spectroscopy (EELS).The interphase regions between GNPs and epoxy matrix was clearly identified by the discrepancy of the plasmon peak positions in the low energy-loss spectra due to different valence electron densities. The spectrum acquisitions were carried out along lines across the interface. An interphase thickness of $13 \mathrm{~nm}$ and $12.5 \mathrm{~nm}$ was measured for GNPs/epoxy and GO/epoxy, respectively. The density of the GNPs/epoxy interphase was $2.89 \%$ higher than that of the epoxy matrix. However, the density of the GO/epoxy interphase was $1.37 \%$ lower than that of the epoxy matrix. The interphase layer thickness measured in this work is in good agreement with the transition layer theory, which proposed an area with modulus linearly varying across a finite width. The results provide an insight into the interphase for carbon-based polymer composites that can help to design the functionalization of nanofillers to improve the composite properties.
\end{abstract}

KEYWORDS: GNPs; GO; Epoxy; Interphase; STEM; EELS; 


\section{INTRODUCTION}

Graphene-based nanocomposites have attracted considerable interest since first reported in $2004^{1-2}$. Benefiting from the outstanding physical and chemical properties of graphene, the multi-functional polymer composites with high performances have been produced by incorporating only a small fraction of graphene in the polymer matrix ${ }^{3-5}$.

However, the final performances of composites reported by different research papers are variable since the properties of nanocomposites depend on a complex set of factors such as dispersion, orientation, interphase properties, etc. Among them, the interphase, an interfacial region which connects chemically or mechanically the nano-fillers and the matrix phases, plays a crucial role to the entire properties of composites ${ }^{6-7}$. For instance, in the epoxy/carbon fiber composites, the interphase transfers the stress from the matrix to nanofillers, which is the key factor to influence the tensile strength ${ }^{8}$. The ways to improve the interphase properties mainly focus on chemical treatments of nano-filler surface so as to introduce active functional groups and also increase physical roughness. Drzal et al ${ }^{9}$ grafted PMMA onto the carbon fiber surface and led to a $25-100 \%$ increase of surface adhesion. Liu et $a l^{10}$ coated thermoplastic poly(phthalazinone ether ketone) (PPEK) on carbon fiber as a sizing agent, which achieved a better thermal stability and compatibility with PPEK resin than the uncoated ones. However, quite differently from the surface treatment of carbon fibers, the chemical treatment of the graphene could cause greatly decrease of the mechanical properties of graphene, e.g. Young's modulus of GO is 207.6GPa compared to $1 \mathrm{TPa}$ for intrinsic graphene ${ }^{11-13}$. Gonçalves et al ${ }^{14}$ compared the mechanical properties of several PMMA composites. The results indicated that with an addition of $1 \%(\mathrm{w} / \mathrm{w})$ of GO-PMMA fillers, the composite had even a smaller Young's modulus than the pure PMMA. Enormous efforts and attempts have been endowed to understand the interphase properties so as to improve interactions and achieve better potential performances of nanocomposites ${ }^{15-18}$. Although numerous works have been published, an in-depth reseach of the interphase, especially a quantitative characterization of the interphase has not been clearly reported yet. The main reason is attributed to the ultra-thin thickness of the interphase ${ }^{19-20}$ and its similar organic components such as the matrix which makes the detecting even more difficult.

Scanning transmission electron microscopy (STEM) combined with electron energyloss spectroscopy is well suitable for acquiring high resolution images with detailed nanostructure information and quantitative analyses on the interphase composition. It has 
been successfully adopted in carbon-based reinforced polymer composites ${ }^{21-23}$. Electron energy-loss spectroscopy (EELS) is an important characterization technique available in most of the transmission electron microscopes. For carbon-based polymer composites, two major regions should be taken into account, the plasmon peak (low-loss region) and the carbon Kedge peak. The former one corresponds to the inelastic interaction of the incident electron beam with the valence-shell electrons, which could be used to obtain the valence electron density of the probed region and to know the physical density of materials; the latter one is the result of the incident beam interaction with the K-shell electrons and reflects the bonding state of the carbon atoms ${ }^{24}$. Leyva-Porras et al. combined STEM and EELS to study the functionalized graphene oxide-reinforced Nylon-6 nanofibers and successfully made a clear distinction between functionalized graphene single-layers, Nylon 6 nanofibers and the carbon substrate $^{25}$. Wu et al. used EELS to focus on the different regions in epoxy/carbon fiber composites. By comparing the plasmon peaks and carbon K-edge, they showed changes in chemical bonding states of different phase regions ${ }^{26}$.

To our best knowledge, as epoxy is a widely used thermoset plastic materials, a quantitative analysis of its interphase with GNPs by STEM/EELS has not been reported in detail. Hence, in this study, we will present a detailed characterization by STEM/EELS analysis of composites reinforced by pristine GNPs and graphene oxide (GO). Focused ion beam (FIB) is employed to prepare TEM samples. The images obtained undeniably reveal the presence of GNPs and GO inside the polymer matrix. EELS analysis, more precisely low-loss peak analysis is presented as a powerful tool to identify the valence electron density in the tested region. Furthermore, the physical density of materials is carried out by using the equations presented in ${ }^{27}$ to have a quantitative evaluation on the interphase density. Interphase thicknesses of different nano-fillers are also compared.

\section{EXPERIMENT}

\section{Materials}

G5, a kind of GNPs, was purchased from KNANO Science Inc., China, and used directly without any other treatment. $\mathrm{KMnO}_{4}(\mathrm{CP}), \mathrm{H}_{2} \mathrm{SO}_{4}(98 \%), \mathrm{HCl}(30 \%)$ and $\mathrm{H}_{2} \mathrm{O}_{2}(30 \%$ solution in water) were purchased from Sigma Aldrich. Epoxy resin (bisphenol F(epichlorhydrin), 1080S) and curing agent (4-4'-methylenebis (cyciohexylamine), 1084) were purchased from Resoltech Ltd., France. 


\section{Sample preparation}

The modified Hummers method was employed for the preparation of GO, as we described elsewhere ${ }^{28}$. The procedure for preparing $1.0 \mathrm{wt} . \%$ G5/Epoxy and GO/Epoxy nanocomposites was as follows: $0.08 \mathrm{~g}$ nanofiller was mixed with $5.94 \mathrm{~g}$ epoxy resin by a three-roll mill (EXAKT 80, Germany) for $30 \mathrm{~min}$. The gap size between the adjacent rollers was set to $50 \mu \mathrm{m}$ and rotation speed was set to $30 \mathrm{rpm}$. Afterwards, $1.98 \mathrm{~g}$ curing agent was added to the collected by manually mixing to obtain a homogeneous suspension. After degassing for 60 min at room temperature in a vacuum oven, the mixture was poured into an aluminum mold with dumbbell-shape which was $1 \mathrm{~mm}$ in thickness, $50 \mathrm{~mm}$ in length and $4 \mathrm{~mm}$ in gage width. Then, the mold was put in an oven at $60^{\circ} \mathrm{C}$ for $10 \mathrm{~h}$.

A FIB column in a Scanning electron microscope (SEM) was used to prepare the sample for the STEM-EELS study. GO/Epoxy and GNPs/Epoxy used for the FIB-SEM was previously immersed in liquid nitrogen for $5 \mathrm{~min}$ and broken fragilely to guarantee the original morphology as in the polymer matrix. The FIB sample preparation was performed in a Helios 660 (FEI) Dual Beam FIB-SEM system with a Ga+ ion source at $30 \mathrm{kV}$. First, the crosssection of the composite was identified and targeted as the region of interest. Then, a $2 \mu \mathrm{m}$ platinum (Pt) protective layer was deposited on the surface of the target milling area, and a coarse and medium milling was performed. A $21 \mathrm{nA}$ beam current was used for the coarse milling until the sample was left with a $2 \mu \mathrm{m}$ thickness, and then $2.5 \mathrm{nA}$ beam current were for medium milling until a thickness of $\simeq 1 \mu \mathrm{m}$ was reached. Afterwards, the section was detached from the surrounding material and transferred to a TEM half-grid for fine thinning. Finally, fine thinning was carried out only at the desired areas until they became as bright as Pt protection layer at $3 \mathrm{kV}$ SEM mode by using $200 \mathrm{pA}$ beam currents, and the amorphous layer on the sample's surface was removed with a low-energy ion beam in three steps, (i) 5 $\mathrm{kV}(41 \mathrm{pA})$, (ii) $2 \mathrm{kV}(23 \mathrm{pA})$ and (iii) $1 \mathrm{kV}(28 \mathrm{pA})$, respectively.

\section{Characterization}

Fourier transform infrared spectra (FT-IR) were recorded on a PE GX spectrometer (Perkin-Elmer, USA) at room temperature from 4000 to $800 \mathrm{~cm}^{-1}$ with a resolution of $4 \mathrm{~cm}^{-1}$. A LabRAM Raman Spectrometer (LabRAM HORIBA Jobin Yvon, Edison, NJ, USA) excited by the $633 \mathrm{~nm}$ coherent line of a He:Ne laser was used to determine the carbon structure of the samples at room temperature; GO was prepared by casting suspensions onto the silicon substrate and drying at $60{ }^{\circ} \mathrm{C}$ for $24 \mathrm{~h}$; GNP powder was directly deposited to the substrate 
before measurement. The microstructure of different surfaces was observed by SEM (ZEISS, LEO 1530 Gemini) at $5 \mathrm{kV}$. TEM imaging was performed using a $\operatorname{Titan}^{3} \mathrm{G} 2$ with a field emission gun (XFEG) operating at an accelerating voltage of $80 \mathrm{kV}$. EELS tests were carried out on the same equipment and operated at $80 \mathrm{kV}$ in scanning transmission electron microscope (STEM) mode. All the images were acquired by high-angle annular dark-field detector. The energy resolution measured with EELS is $0.8 \mathrm{eV}$. Digital images and energyloss spectra were captured using an Enfinium ER filter with a dispersion of $0.1 \mathrm{eV} / \mathrm{channel}$. The spectral background was removed by fitting the pre-edge background with a power-law function. Obtaining spectra in the STEM mode using a camera length of $115 \mathrm{~mm}$ and a spectrometer entrance aperture diameter of $5 \mathrm{~mm}$ provided a collection semi-angle $(\boldsymbol{\beta})$ of 14.7 mrad. Convergence angle $(\boldsymbol{\alpha})$ was about $16.7 \mathrm{mrad}$. Further spectral processing operations were conducted using digital micrograph (DM) software.

\section{RESULTS and DISCUSSION}

\subsection{GO preparation}

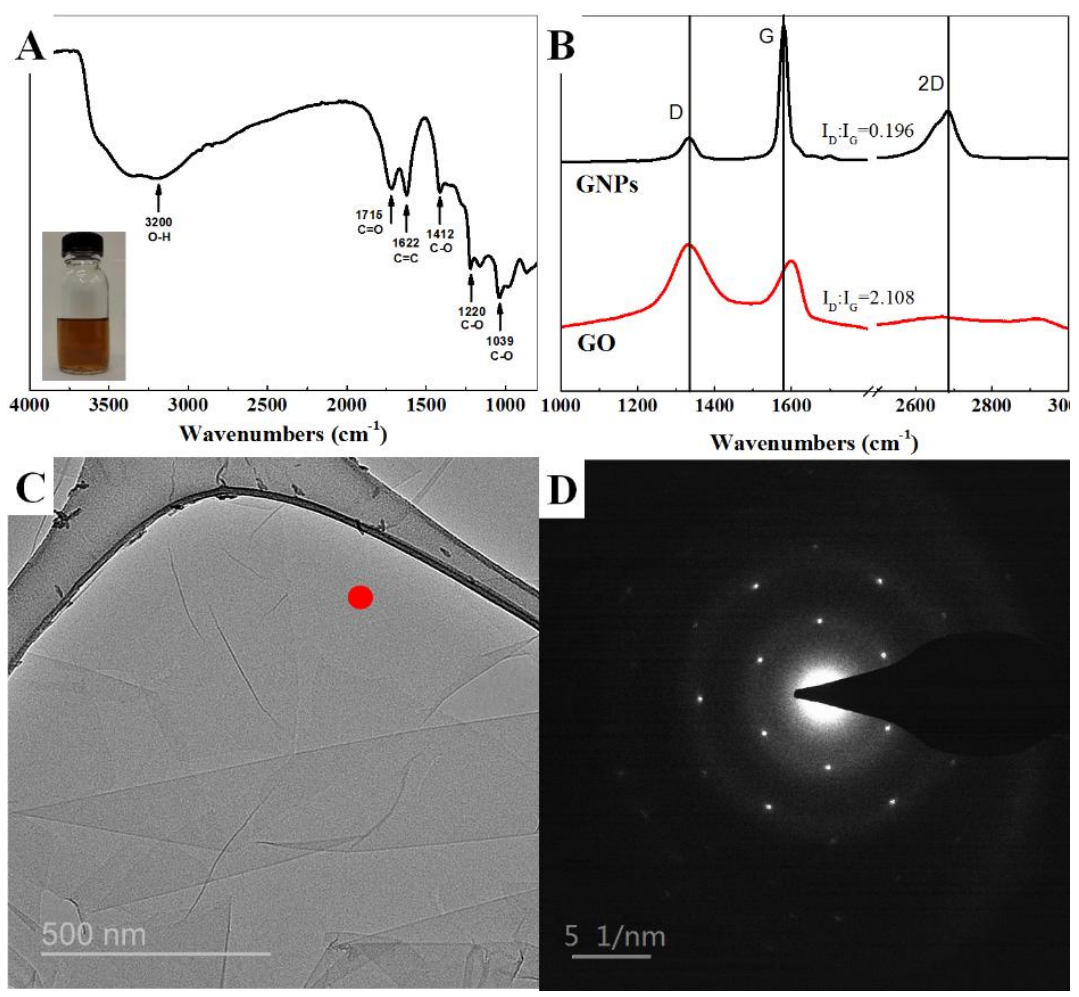

Fig. 1 (A) FT-IR curve of GO; the inserted image is the GO dispersed in ethanol by ultrasonication; (B)

Raman spectra of G5 and GO; (C) TEM image of GO; (D) selected area diffraction pattern in the position of red point in (C). 
GO was prepared by the same type of GNPs, which is ultra-thin and semi-transparent under SEM as showed in Fig. S1. Fig. 1A shows the FT-IR curves of GO. For GO, the oxidation in sulfuric acid provided a typical absorption peak at $1715 \mathrm{~cm}^{-1}$ associated with the carboxyl group and also an absorbency at approximately $1039 \mathrm{~cm}^{-1}$-which corresponds to the other side groups. Other oxygen-containing groups were also marked out in the spectra ${ }^{29-30}$.

In the Raman spectra shown in Fig. 1B, the G5 has a weak D band at $1350 \mathrm{~cm}^{-1}$ and an incisive $\mathrm{G}$ band at $1580 \mathrm{~cm}^{-1}$. However, the D band of GO strongly increases while the $\mathrm{G}$ band shifts to higher wave numbers due to the graphite amorphization, which confirms the lattice distortions caused by oxidation. We also employed the ratio between $D$ and $G(D / G)$ to roughly estimate the oxidation degree of GO. In the case of G5, this value is as low as 0.196. While in the case of GO, this value increases to 2.108 . Furthermore, the $2 \mathrm{D}$ band at $2700 \mathrm{~cm}^{-1}$ is highly sensitive to the stacking of graphene sheets ${ }^{31}$. The Raman spectrum of G5 shows an incisive band related to its few-layer structure. After oxidation, GO shows an obviously broadened peak with a lower intensity around $2700 \mathrm{~cm}^{-1}$.

Fig. 1(C) shows the TEM image of GO. After ultrasonication, most of GO platelets were dispersed to the monolayer's state. We chose a zone to obtain the selected area diffraction pattern and it can be found that a portion of GO still maintains the structure of the graphene due to the hexagon pattern shown in the Fig. 1(D); at the same time, the appearance of the bright circles indicates the amorphization of graphene structure after oxidation.

\subsection{G5 \& GO/epoxy nanocomposites}



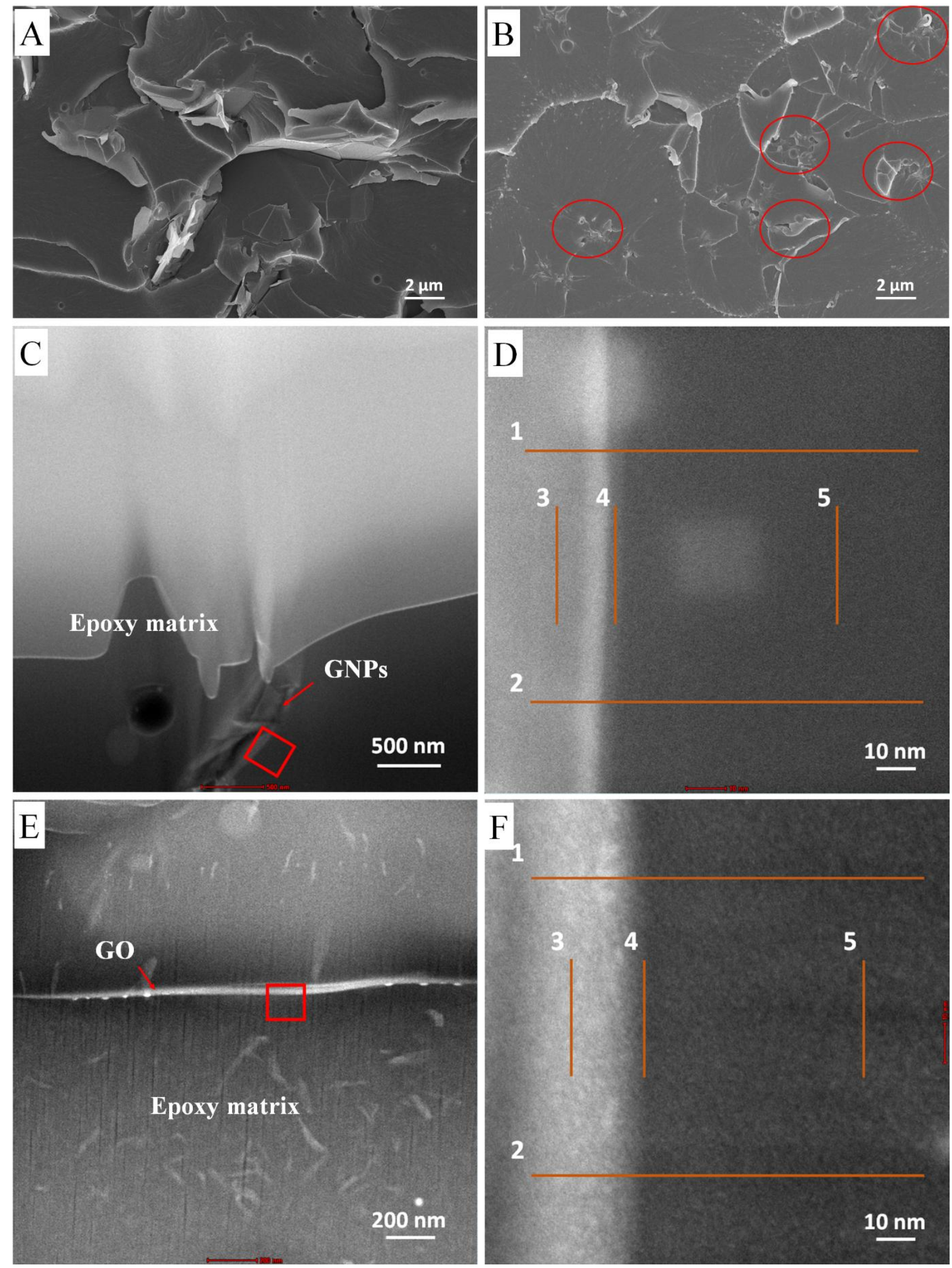

Fig. 2 SEM images of (A) the G5/epoxy and (B) the GO/epoxy fracture surface with $1.0 \%$ weight fraction. Overview of STEM Z-contrast image showing: (C) a GNP plane perpendicular to the epoxy foil prepared by FIB, (E) the GO/epoxy sample. (D) and (F) show higher magnification Z-contrast images of highlight regions in (C) and (E), respectively. 
In the SEM images of G5/epoxy (A) and GO/epoxy (B) nano-composites with $1.0 \mathrm{wt} \%$ shown in Fig.2, it can be found that after incorporation into the polymer matrix, the G5 are homogeneously distributed. Besides, some GNPs are pulled out directly from the epoxy matrix. It may infer that the adhesion between GNPs and PMMA is not very strong due to non-covalent bonding. For GO/Epoxy nano-composite as shown in the (B), there exists some aggregations instead of the entire platelet shape of GO, as marked out by red circles. GO and epoxy has similar components, which are difficult to be recognized by SEM. The existing oxygen-containing groups on the GO surface enable to enhance the interaction with epoxy matrix. Furthermore, after oxidation, the graphene size has decreased.

Fig. 2(C) presents a STEM image of G5/Epoxy, in which the bright region in the upper side shows the pure epoxy with larger thickness due to the inappropriate thinning process during sample preparation. At the bottom, the relatively thin region contains the G5 platelet. The in-plane of GNPs is perpendicular to the sample foil. Both sides of the GNPs' edges adhere to the matrix, and show a bright grey color. In the thin region, the epoxy shows the same contrast indicating the uniform thickness in this part.

Fig. 2(E) shows a STEM image of GO/Epoxy. During three-roll process, some single layer GO have been broken into some small pieces. In the image, some homogeneously distributed bright parts indicate the small GO pieces. However, they are not perpendicular to the sample plane. The bright line appearing in the image is the aggregation of GO layers, which are vertical to the plane and more suitable for the interface study.

Two regions in Fig. 2(C) and 2(E) are enarged: Fig. 2(D) and Fig. 2(F) correspond to the red rectangular region in Fig. 2(C) and Fig. 2(E), respectively. First, in Fig. 2(D), the dark region is the epoxy matrix while the bright one is GNPs. In high resolution, some surface bulges could be observed in the region. In the interface, the boundary is obvious and smooth, the brighter region in GNPs side and grey region in the matrix part compose a transition layer, namely the interphase. Meanwhile, we acquire EELS spectra in the positions marked by five orange lines, two horizontal lines with length of $100 \mathrm{~nm}$ and three vertical lines with length of $30 \mathrm{~nm} .100$ spectra were acquired on each horizontal line and 30 spectra on each vertical line leading to an average acquisition distance between two points of $0.5 \mathrm{~nm}$. Secondly, Fig. 2(F) is the enlargement of the red rectangle in Fig. 2(E). There exist two parts, the GO and the epoxy matrix. The boundary is fuzzy and there exists a region changing continuously, which is due to the better connection between GO and epoxy matrix. The hydroxyl and epoxy 
groups on the GO plane have chemical reaction with the curing agent and covalently bond to the epoxy net. Then, the same EELS acquisition process has been conducted at the interphase region.

\subsection{Interphase characterization}
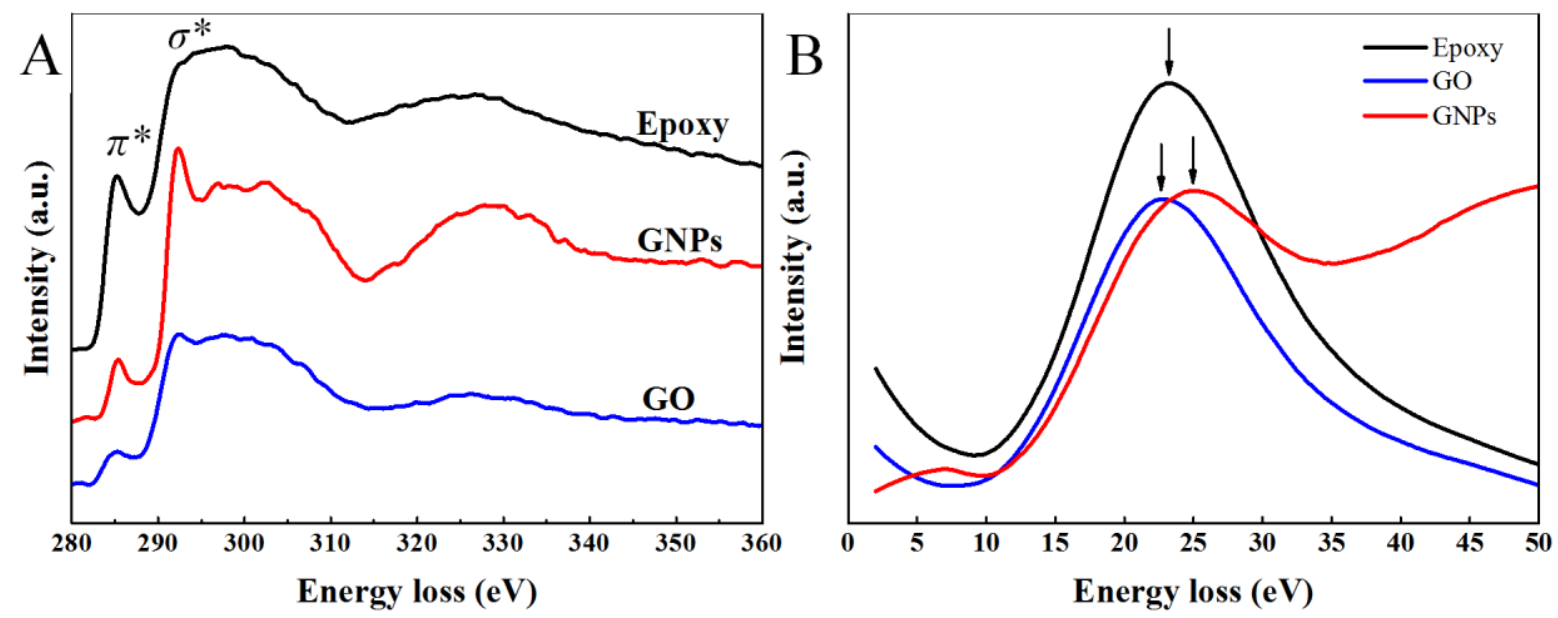

Fig. 3 (A) Carbon core-loss and (B) low-loss spectra acquired from epoxy matrix, GNPs and GO, respectively.

Electron energy loss spectroscopy (EELS) is currently the preferred method to obtain the $s p^{3}$ content from the size of the $\pi^{*}$ peak in the carbon K-edge absorption spectrum, where the low energy loss spectrum gives the valence plasmon energy and thereby the mass density.

Normalized EEL spectra showing the carbon core-loss and low-loss are presented in Fig. 3. In Fig. 3(A), it is possible to observe the differences between the response intensity of the edges and the shape of the spectral curves. At an energy loss of $285.3 \mathrm{eV}$, the $1 \mathrm{~s}$ to $\pi^{*}$ transition is observed, while the states corresponding to a transition from $1 s$ to $\sigma^{*}$ were observed at $292 \mathrm{eV}^{32-33}$. These transitions are the main features of the EEL spectra for graphite and other carbon-based materials and both correspond to the excitation fingerprint of the valence band electrons, above the Fermi level. Identification of the transitions in EEL spectra is done by carefully observing each of the spectra. The only method employed during this task is the background subtraction and the plural scattering removal. Once these procedures are applied, spectra are observed similar to those presented in Fig. 3(A). From the detailed observation of each spectrum, GNPs have a narrow and incisive $\pi^{*}$ transition compared with the two other spectra. After oxidation, the $\pi^{*}$ transition of GO is still 
noticeable, but with a reduced intensity. However, for the epoxy matrix, the $\pi^{*}$ transition has been already immersed to some low-signal peaks.

In the low-loss spectrum, a broad peak appears in the range from $22 \mathrm{eV}$ to $25 \mathrm{eV}$. Comparing the low-loss spectra of GNPs with those of epoxy, it has been found that the lowloss peak of GNPs shifts towards higher energy-loss region than that of epoxy. However, the low-loss peak of GO shifts towards lower energy-loss region comparing with that of epoxy. The arrows point out the low-loss peak, which correspond to the excitation of $\pi+\sigma$ electron in the polyaromatic solid. This peak position is identified as $E_{p}{ }^{34}$.

According to the expression relating the plasmon energy to the valence electron density, e.g. ${ }^{27}$,

$$
E_{p}=\hbar\left(\frac{n_{e} e^{2}}{\varepsilon_{0} m^{*}}\right)^{\frac{1}{2}}
$$

where $n_{e}$ stands for the valence electron density, $\varepsilon_{0}$ for the vacuum dielectric function, $m^{*}$ for the electron effective mass, and $m$ for the free electron mass, respectively.

The mass density is derived from the valence electron density $n_{e}$ by assuming that carbon contributes with four valence electrons, oxygen with six and hydrogen with one, to obtain

$$
n_{e}=12 \frac{\rho N_{A}}{M_{C}}\left(\frac{3 X_{C}+5 X_{O}+1}{11 X_{C}+15 X_{O}+1}\right)
$$

With $X_{H}=1-X_{C}-X_{O}$. Where $X_{H}, X_{C}$ and $X_{O}$ correspond to the element fraction of hydrogen, carbon and oxygen, respectively. Thus, the mass density is given by

$$
\rho=\frac{\varepsilon_{0}}{12 \hbar^{2} N_{A} e^{2}} M_{C} m^{*} E_{P}^{2}\left(\frac{11 X_{C}+15 X_{O}+1}{3 X_{C}+5 X_{O}+1}\right)
$$

Thus, for the same chemical component region, a high value of $E_{p}$ usually leads to a high density. In the epoxy matrix, the mass density can be compared by

$$
\rho \propto E_{p}{ }^{2}
$$

To study the physical density of the interface region, we have done linear acquisition across the interface region. In Fig. 2 (D) and (F), we have already acquired 100 low energy loss spectra for each horizontal line and 30 spectra for each vertical line, respectively. After smoothing treatment of spectra by origin, the peak position value of each spectrum can be 
extracted. Then, for each region, we put all the values as a function of acquired positions which are presented in Fig.4. The points are counted from left to right side for horizontal lines and up to bottom for vertical lines.

Figure 4(A) corresponds to the line 1 in Fig. 2(D), in which we can easily distinguish the GNPs region and epoxy region since the average energy loss of GNPs is higher than that of epoxy matrix, $24.86 \pm 0.135 \mathrm{eV}$ for GNPs and $23.70 \pm 0.08 \mathrm{eV}$ for epoxy matrix, respectively. Between them, there exists a narrow region in which the energy-loss decreased gradually. Referring to the STEM image of Fig. 2(D), this region contains two parts, a GNP region from point 11 to point 19 , and an epoxy region from point 20 to point 24 , which is considered as transition layer, also namely interphase, as we marked out by $\mathbf{1}$ and $\mathbf{2}$, respectively. As mentioned before, the distance between each point is $1 \mathrm{~nm}$ and thus the thickness of the interphase is $14 \mathrm{~nm}$. Then, we calculate the average $E_{p}$ value of epoxy in interphase and matrix region: they are $23.95 \pm 0.176 \mathrm{eV}$ for epoxy in region 2 and $23.70 \pm 0.08 \mathrm{eV}$ for the epoxy matrix, respectively. By using the equation (4), it can be found clearly that the density of epoxy in the interphase region has increased by $2.12 \%$ compared with the matrix. If one of the evaluations for the reinforcement of nanofillers are supposed to be the increase of the density ${ }^{35}$, it is obvious that GNPs have the enhancement to the surrounded polymer matrix. 
A

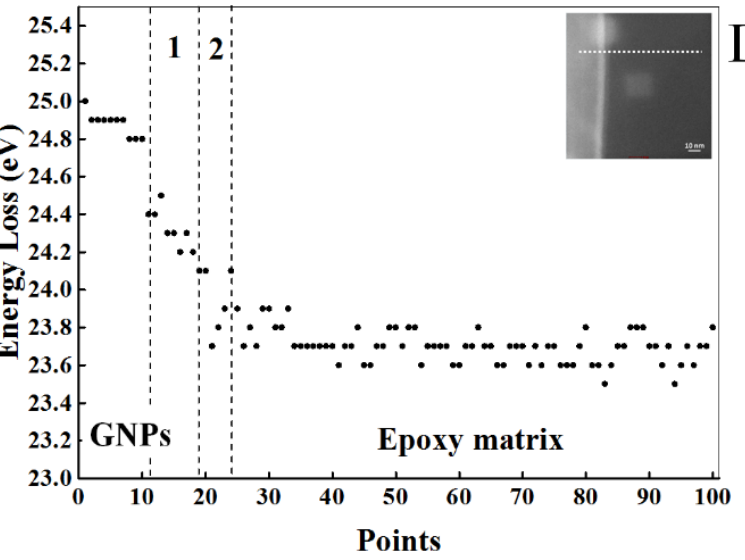

$\mathrm{B}$

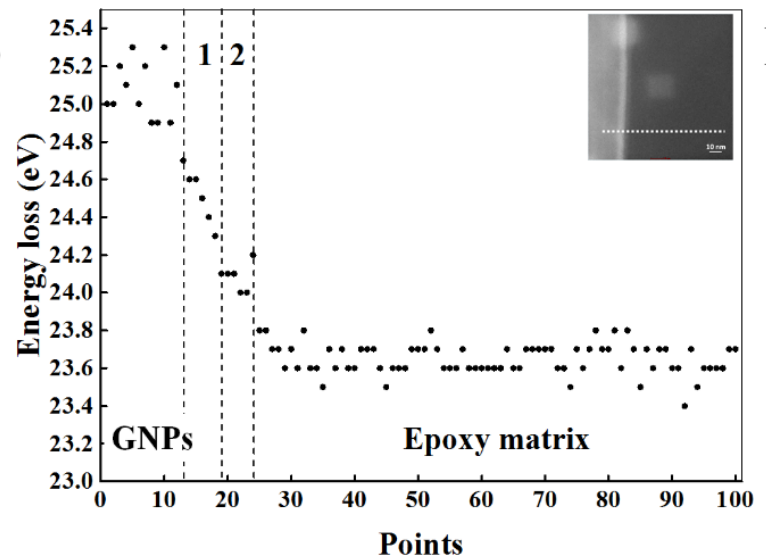

C

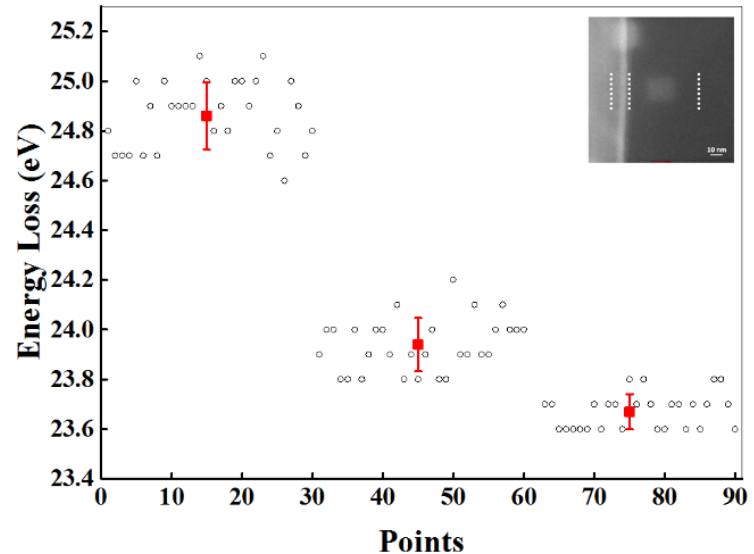

D

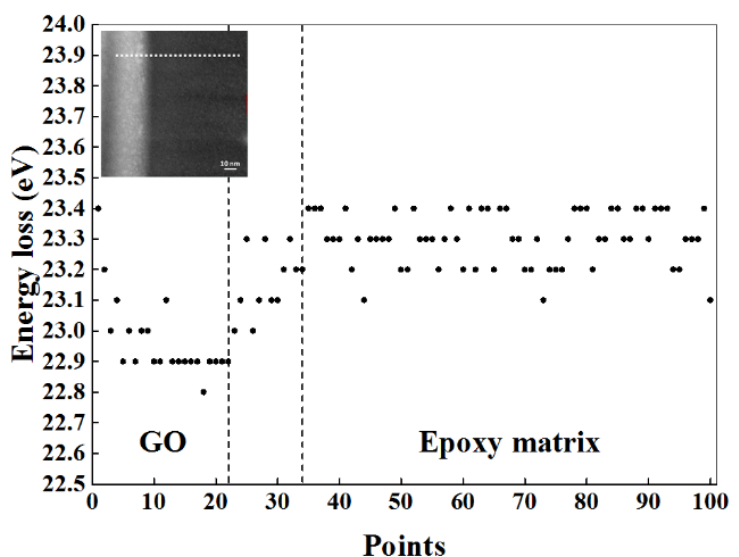

E

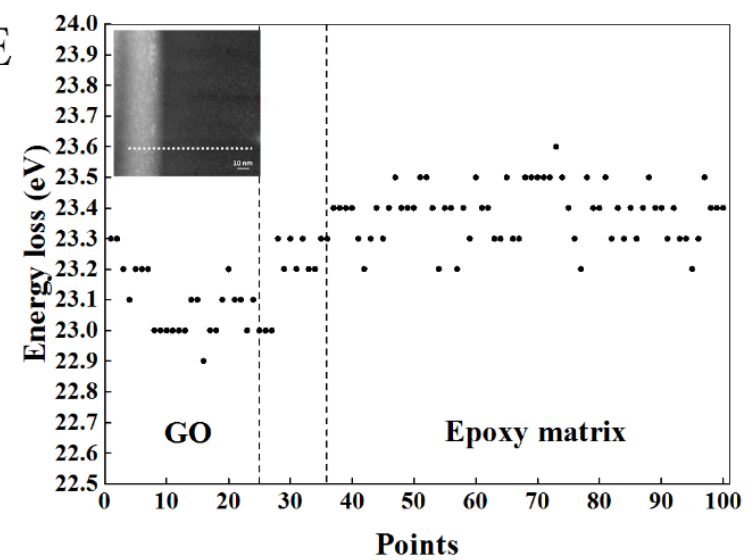

$\mathrm{F}$

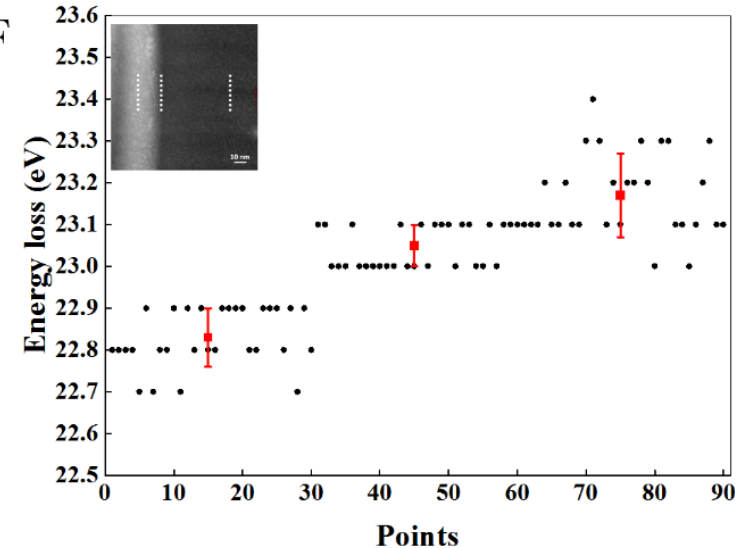

Fig. 4 the peak positions extracted from the low energy-loss spectra acquired from Fig. 2(D) and (F), shown as a function of the acquisition order from point 1 to point 100: (A) for line 1 in Fig. 2(D); (B) for line 2 in Fig. 2(D); (D) for line 1 in Fig. 2(F); (E) for line 2 in Fig. 2(F); three vertical lines in each images showed the low energy loss spectra acquisition from three regions, GNPs, epoxy in interphase region and epoxy matrix; the numbers indicate the acquisition order during experiment, point 1 to point 30 for line 3, point 31 to point 60 for line 4 and point 61 to point 90 for line 5, respectively; (C) 90 peak position values of low energy loss spectra from Fig. 2(D); (F) 90 peak position values of low energy loss spectra from Fig. 2(F). The insect images indicate the acquisition position for each spectra.

(All the peak values are listed in the table S1) 
To verify the enhancement phenomenon, as showed in Fig. 2(D), another region is selected to acquire low energy-loss spectra and treated by the same way. The extracted value is shown in Fig. 4(B). In the graph, three regions can be obviously distinguished which correspond to the GNPs, the epoxy in the interphase region and the epoxy matrix, respectively. The average energy-loss value of three regions are $24.86 \pm 0.135 \mathrm{eV}, 24.08 \pm 0.075 \mathrm{eV}$ and $23.65 \pm 0.08 \mathrm{eV}$, respectively. These values are similar to the former one. After the comparison, it can be found that the density of epoxy in the interphase region has been improved by $3.67 \%$ with the thickness of $12 \mathrm{~nm}$. For the same nanofillers, the enhancements on the density and thickness of the interphase almost keep at the same level.

To make a comparison with the GNPs, we also acquire the low energy-loss spectra of GO/Epoxy interface. An aggregated GO platelet was chosen for the acquisition of EEL spectra since single layer GO is too small and rarely perpendicular to the foil plane. 100 spectra are acquired in the interface region with the length of $100 \mathrm{~nm}$. The same procedure is done to the acquired low energy-loss spectra. We obtain the peak position value and present it in Fig. 4(D) and (F). Referring to Fig. 2(F), the situation is reversed, i.e. the $E_{p}$ of GO is lower than that of the epoxy matrix, $22.83 \pm 0.07 \mathrm{eV}$ for $\mathrm{GO}$ and $23.17 \pm 0.10 \mathrm{eV}$ for the epoxy matrix, respectively. It is reasonable since GO has almost the same density as the epoxy matrix. After oxidation, the interlamellar space of GO is doubled compared with graphite. Using this approach, the density of GO has been halved $\left(\rho=1.06 \mathrm{~g} / \mathrm{cm}^{3}\right)^{5}$. However, there still exists a transition region between GO and the epoxy matrix. As marked by a dashed line in Fig. 4(D) and (F), the average thickness of the transition layer is $12.5 \mathrm{~nm}$. Due to the fuzzy GO/Epoxy boundary, the entire transition layer is considered as the interphase region. After calculation, in both lines, the values show the interphase region with the decrease density of $1.11 \%$ and $1.62 \%$ comparing with the epoxy matrix, respectively. Furthermore, the $E_{p}$ of GO/Epoxy composite is decreased by $0.5 \mathrm{eV}$ when compared with that of GNPs/Epoxy. This could be due to the oxygen-containing groups on the GO surface. These groups could have competition with the resin to react with the curing agent, leading to a small decrease of the matrix density.

To further confirm the stability of the interphase, the line acquisitions are conducted in the same region as showed Fig. 2(D) and (F). Three vertical lines are set at the GNP (GO), the epoxy interphase region and the matrix with a length of $30 \mathrm{~nm}$, respectively. 30 spectra are acquired from each line. The data are treated by the same procedure as discussed before. The peak position values are presented in Fig. $4(\mathrm{C})$ and $(\mathrm{F})$ as a function of the acquisition order. In Fig. 4(C), it is obvious that from GNPs to epoxy region, the value of $E_{p}$ decreases gradually: 
$24.86 \pm 0.135 \mathrm{eV}$ for GNPs, $23.94 \pm 0.107 \mathrm{eV}$ for epoxy in the interphase region and $23.67 \pm 0.071 \mathrm{eV}$ for epoxy matrix, respectively. The situation is reversed in Fig. 4(F), in which the value of $E_{p}$ increases gradually from GO to the epoxy matrix: $22.83 \pm 0.07 \mathrm{eV}$ for GO, $23.05 \pm 0.05 \mathrm{eV}$ for epoxy in the interphase region and $23.20 \pm 0.10 \mathrm{eV}$ for the epoxy matrix, respectively.

A

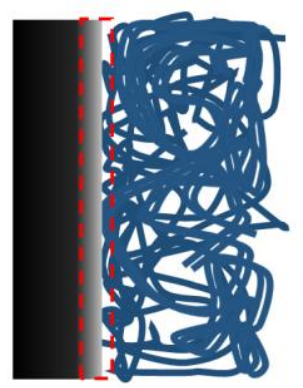

GNPs $\quad \stackrel{\mathbf{r}-\mathbf{I}}{-}$ Interphase region

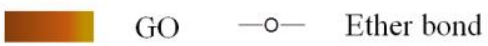

C

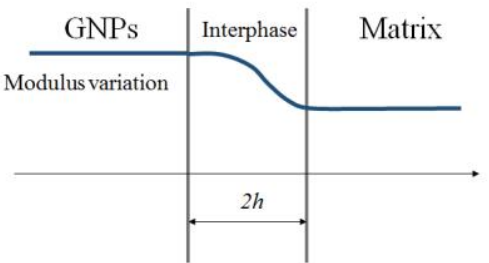

B

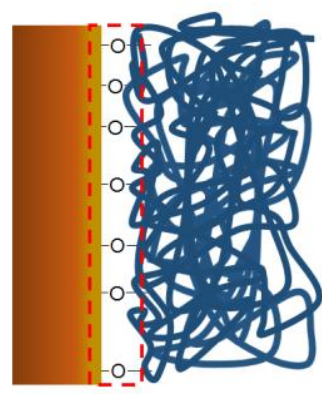

$=$ Matrix

D

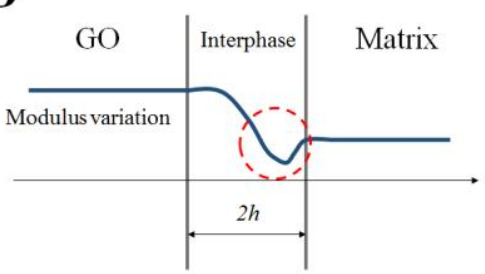

Fig. 5 Schematic representation of a composite interphase: (A) GNPs/Epoxy, (B) GO/Epoxy; modulus variation in the interphase: (C) GNPs/Epoxy and (D) GO/Epoxy.

According to our results, there exists an interphase region, which connects the reinforced part and the polymer matrix. Due to the crosslinking, the epoxy matrix forms a net structure surrounding the nanofillers. In the case of GNPs, due to its chemical inertness, no chemical reaction happened in the interphase region, leading to a clear and smooth interface, as shown in Fig. 5(A). After oxidation, the oxygen-containing groups have been introduced onto the graphene plane, epoxyl groups on the plane can react with the curing agent, as shown in Fig. 5(B). The boundary between GO and epoxy is blurred. The density of GO also halved due to the intermellar enlargement of graphite.

The transition layer where the elastic moduli vary continuously between those of the two materials has been discussed by Delale and Erdogan in $1988^{36}$. They considered the thickness of this transition layer as $2 h$, ranging from nanometers for an atomistically sharp 
interface, to micrometers for a diffused bimaterial interface. Based on our work, it has been found that this transition layer is composed of two parts, the nanofiller and the polymer, respectively. Those with a high density part take up a thicker transition region, as shown before, $8 \mathrm{~nm}$ GNPs part in 13 transition layer of GNPs/Epoxy. The larger difference between the density of two component, the thicker thickness exists.

Based on Fan's work ${ }^{35}$, there exist a rough relationship between the modulus and the

density, $\frac{E_{1}}{E_{2}} \sim\left(\frac{\rho_{1}}{\rho_{2}}\right)^{2}$. For the same type of material, a higher density leads to a larger modulus. In the low-loss spectra, the density decreased from GNPs to epoxy matrix in the transition layer, indicating the decreasing of the modulus from nanofillers to matrix, as shown in Fig. 5(C). For GO/Epoxy composites, the situation is reversed, in which the matrix has a higher density than that of GO. Although GO has a much lower modulus than the original GNPs, its modulus is still higher than that of the Epoxy matrix. Thus, there exists a minimum modulus, which must have a negative influence on the entire properties of the final composites, as marked out by the red circle in Fig. 5(D).

To obtain better properties of nanocomposites, the modification of nanofillers is usually taken into account since an improved interphase/interface can be achieved between the nanofillers and matrix. However, for Graphene-based composites after the modification, more defects could be introduced which can negatively influence their intrinsic properties. Although the chemical bonds exist at the interface, the entire mechanical properties of GO/polymer decrease compared with GNPs/Epoxy. Therefore, a compromise should be considered before functionalization/modification.

\section{CONCLUSION}

In this work, we quantitatively studied the interphase structures between epoxy matrix and graphene-based materials by using STEM and EELS. By analyzing the low energy-loss spectra, we have successfully identified the interphase zone. There exists a transition layer between polymer matrix and nanofillers, which bridges two parts with a gradually changed density. The density of the GNPs/epoxy interphase has been found to be $2.89 \%$ higher compared with that of the epoxy matrix. However, the density of the GO/epoxy interphase has decreased by $1.37 \%$ compared with the epoxy matrix. While many oxygen-containing groups were introduced onto the graphene surface after oxidation, the intrinsic properties of graphene sheets have been decreased largely. This work gives an understanding of the interphase of 
carbon-based composites and also provides an indication for the effects of the modification of nanofillers. A better interaction between the nanofillers and the matrix in the interface leads to efficient energy transfer; while the excellent properties of nanofillers should be maintained. Those two aspects should be considered in fabricating polymer mtrix composites.

\section{Supporting Information}

This material includes the detailed peak values of Figure 4 and a SEM image of original GNPs.

\section{ACKNOWLEDGMENTS}

This work was carried out within the MATMECA consortium and supported by the ANR under contract number ANR-10-EQPX-37. It has benefited from the facilities of the Laboratory MSSMat (UMR CNRS 8579), CentraleSupélec, France. The authors thank Dr. H. ZHAO for the experiment discussion. Yu Liu thanks China Scholarship Council for the fellowship. The GNPs used in this paper was purchased by GDR 3661 Polynano.

\section{REFERENCES}

(1) Novoselov, K. S.; Geim, A. K.; Morozov, S. V.; Jiang, D.; Zhang, Y.; Dubonos, S. V.; Grigorieva, I. V.; Firsov, A. A., Electric Field Effect in Atomically Thin Carbon Films. science 2004, 306, 666-669.

(2) Geim, A. K.; Novoselov, K. S., The Rise of Graphene. Nat Mater 2007, 6, 183-191.

(3) Yang, X.; Li, L.; Shang, S.; Tao, X.-m., Synthesis and Characterization of Layeraligned Poly (vinyl alcohol)/Graphene Nanocomposites. Polymer 2010, 51, 3431-3435. (4) Qi, X.-Y.; Yan, D.; Jiang, Z.; Cao, Y.-K.; Yu, Z.-Z.; Yavari, F.; Koratkar, N., Enhanced Electrical Conductivity in Polystyrene Nanocomposites at Ultra-low Graphene Content. ACS Appl. Mater. Interfaces 2011, 3, 3130-3133.

(5) Rafiee, M. A.; Rafiee, J.; Wang, Z.; Song, H.; Yu, Z.-Z.; Koratkar, N., Enhanced Mechanical Properties of Nanocomposites at Low Graphene Content. ACS nano 2009, 3, 3884-3890.

(6) Kuilla, T.; Bhadra, S.; Yao, D.; Kim, N. H.; Bose, S.; Lee, J. H., Recent Advances in Graphene Based Polymer Composites. Prog. Polym. Sci. 2010, 35, 1350-1375.

(7) Sengupta, R.; Bhattacharya, M.; Bandyopadhyay, S.; Bhowmick, A. K., A Review on the Mechanical and Electrical Properties of Graphite and Modified Graphite Reinforced Polymer Composites. Prog. Polym. Sci. 2011, 36, 638-670. 
(8) Tang, L. G.; Kardos, J. L., A Review of Methods for Improving the Interfacial Adhesion Between Carbon Fiber and Polymer Matrix. Polym. Compos. 1997, 18, 100-113.

(9) Drzal, L.; Raghavendran, V., Adhesion of Thermoplastic Matrices to Carbon Fibers: Effect of Polymer Molecular Weight and Fiber Surface Chemistry. J. Thermoplast. Compos. Mater. 2003, 16, 21-30.

(10) Liu, W.; Zhang, S.; Hao, L.; Jiao, W.; Yang, F.; Li, X.; Wang, R., Properties of Carbon Fiber Sized with Poly (Phthalazinone Ether Ketone) Resin. J. Appl. Polym. Sci. 2013, $128,3702-3709$.

(11) Frank, I. W.; Tanenbaum, D. M.; van der Zande, A. M.; McEuen, P. L., Mechanical Properties of Suspended Graphene Sheets. J. Vac. Sci. Technol., B 2007, 25, 2558-2561.

(12) Lee, C.; Wei, X.; Kysar, J. W.; Hone, J., Measurement of the Elastic Properties and Intrinsic Strength of Monolayer Graphene. Science 2008, 321, 385-388.

(13) Suk, J. W.; Piner, R. D.; An, J.; Ruoff, R. S., Mechanical Properties of Monolayer Graphene Oxide. ACS nano 2010, 4, 6557-6564.

(14) Gonçalves, G.; Marques, P. A.; Barros-Timmons, A.; Bdkin, I.; Singh, M. K.; Emami, N.; Grácio, J., Graphene Oxide Modified with PMMA via ATRP as A Reinforcement Filler. $J$. Mater. Chem. 2010, 20, 9927-9934.

(15) Zaman, I.; Phan, T. T.; Kuan, H.-C.; Meng, Q.; La, L. T. B.; Luong, L.; Youssf, O.; Ma, J., Epoxy/Graphene Platelets Nanocomposites with Two Levels of Interface Strength. Polymer 2011, 52, 1603-1611.

(16) Yu, A.; Ramesh, P.; Itkis, M. E.; Bekyarova, E.; Haddon, R. C., Graphite Nanoplatelet-Epoxy Composite Thermal Interface Materials. J. Phys. Chem. C 2007, 111, 7565-7569.

(17) Ma, J.; Meng, Q.; Michelmore, A.; Kawashima, N.; Izzuddin, Z.; Bengtsson, C.; Kuan, H.-C., Covalently Bonded Interfaces for Polymer/Graphene Composites. J. Mater. Chem. A 2013, $1,4255-4264$.

(18) Desai, A.; Haque, M., Mechanics of the Interface for Carbon Nanotube-Polymer Composites. Thin Wall. Struct. 2005, 43, 1787-1803.

(19) Gao, S.-L.; Mäder, E.; Zhandarov, S. F., Carbon Fibers and Composites with Epoxy Resins: Topography, Fractography and Interphases. Carbon 2004, 42, 515-529.

(20) Drzal, L. T.; Rich, M. J.; Koenig, M. F.; Lloyd, P. F., Adhesion of Graphite Fibers to Epoxy Matrices: II. The Effect of Fiber Finish. J Adhes. 1983, 16, 133-152.

(21) Warner, J. H.; Lin, Y.-C.; He, K.; Koshino, M.; Suenaga, K., Atomic Level Spatial Variations of Energy States along Graphene Edges. Nano Lett. 2014, 14, 6155-6159. 
(22) Zhou, W.; Lee, J.; Nanda, J.; Pantelides, S. T.; Pennycook, S. J.; Idrobo, J.-C., Atomically Localized Plasmon Enhancement in Monolayer Graphene. Nat. Nanotechnol. 2012, 7, 161-165.

(23) Allen, L.; Findlay, S.; Lupini, A.; Oxley, M.; Pennycook, S., Atomic-Resolution Electron Energy Loss Spectroscopy Imaging in Aberration Corrected Scanning Transmission Electron Microscopy. Phys. Rev. Lett. 2003, 91, 105503.

(24) Egerton, R. F., Electron Energy-Loss Spectroscopy in the Electron Microscope, third edition. Springer Science \& Business Media 2011, 306-333.

(25) Leyva-Porras, C.; Ornelas-Gutiérrez, C.; Miki-Yoshida, M.; Avila-Vega, Y. I.; Macossay, J.; Bonilla-Cruz, J., EELS Analysis of Nylon 6 Nanofibers Reinforced with Nitroxide-Functionalized Graphene Xxide. Carbon 2014, 70, 164-172.

(26) Wu, Q.; Li, M.; Gu, Y.; Li, Y.; Zhang, Z., Nano-Analysis on the Structure and Chemical Composition of the Interphase Region in Carbon Fiber Composite. Composites, Part A 2014, 56, 143-149.

(27) Ferrari, A. C.; Libassi, A.; Tanner, B. K.; Stolojan, V.; Yuan, J.; Brown, L. M.; Rodil, S. E.; Kleinsorge, B.; Robertson, J., Density, $s p^{3}$ Fraction, and Cross-Sectional Structure of Amorphous Carbon Films Determined by X-ray Reflectivity and Electron Energy-Loss Spectroscopy. Phys. Rev. B 2000, 621, 11089-11103.

(28) Liu, Y.; Zhang, Y.; Zhang, T.; Jiang, Y.; Liu, X., Synthesis, Characterization and Cytotoxicity of Phosphorylcholine Oligomer Grafted Graphene Oxide. Carbon 2014, 71, 166175.

(29) Stankovich, S.; Piner, R. D.; Nguyen, S. T.; Ruoff, R. S., Synthesis and Exfoliation of Ssocyanate-treated Graphene Oxide Nanoplatelets. Carbon 2006, 44, 3342-3347.

(30) Marcano, D. C.; Kosynkin, D. V.; Berlin, J. M.; Sinitskii, A.; Sun, Z.; Slesarev, A.;

Alemany, L. B.; Lu, W.; Tour, J. M., Improved Synthesis of Graphene Oxide. ACS nano 2010, 4, 4806-4814.

(31) Ferrari, A.; Meyer, J.; Scardaci, V.; Casiraghi, C.; Lazzeri, M.; Mauri, F.; Piscanec, S.; Jiang, D.; Novoselov, K.; Roth, S., Raman Spectrum of Graphene and Graphene Layers. Phys. Rev. Lett. 2006, 97, 187401.

(32) Ponsonnet, L.; Donnet, C.; Varlot, K.; Martin, J. M.; Grill, A.; Patel, V., EELS Analysis of Hydrogenated Diamond-like Carbon Films. Thin Solid Films 1998, 319, 97-100. (33) Rosenberg, R. A.; Love, P. J.; Rehn, V., Polarization-Dependent C K Near-edge X-ray Absorption Fine Structure of Graphite. Phys. Rev. B 1986, 33, 4034-4037. 
(34) Laffont, L.; Monthioux, M.; Serin, V., Plasmon as A Tool for in-situ Evaluation of Physical Properties for Carbon Materials. Carbon 2002, 40, 767-780.

(35) Fan, H.; Hartshorn, C.; Buchheit, T.; Tallant, D.; Assink, R.; Simpson, R.; Kissel, D. J.; Lacks, D. J.; Torquato, S.; Brinker, C. J., Modulus-Density Scaling Behaviour and Framework Architecture of Nanoporous Self-asembled Silicas. Nat. Mater. 2007, 6, 418-423.

(36) Delale, F.; Erdogan, F., On the Mechanical Modeling of the Interfacial Region in Bonded Half-planes. J. Appl. Mech. 1988, 55, 317-324. 